\title{
Prevalence and extent of right-to-left shunt on contrast- -enhanced transcranial Doppler in patients with chronic hyperventilation syndrome: results of a case-control study
}

\author{
Jacek Staszewski, Kazimierz Tomczykiewicz, Anna Piusińska-Macoch, Adam Stępień \\ Clinic of Neurology, Military Medical Institute, Szaserow 128, 04-141 Warsaw, Poland
}

\begin{abstract}
Aim. Chronic hyperventilation syndrome (CHVS) represents a frequent but poorly understood breathing pattern disorder. In a previous small pilot study, we reported a higher prevalence of right-to-left shunt (RLS) in CHVS patients than in healthy subjects. The aim of this study was to confirm those previous results from this larger and matched case-control study, and to evaluate the prevalence and grade of RLS in patients with CHVS in whom organic and psychiatric causes were excluded.

Clinical rationale for the study. Determining other types of CHVS triggers not related to organic or psychiatric causes which could be clinically useful.

Material and methods. 100 subjects (mean age $34 \pm 6$ years; $80 \%$ females), including 50 patients with CHVS and 50 age- and sex-matched healthy controls (CG), were prospectively recruited into this single-centre study. Vascular RLS was diagnosed using contrast-enhanced transcranial Doppler (C-TCD).

Results. RLS prevalence significantly increased in the CHVS group $(n=23)$ compared to the CG group $(n=8)(46 \%$ versus $16 \%$; $p<0.01)$. Patients with CHVS and RLS tended to have more frequent permanent shunts compared to the CG $(60 \%$ versus $25 \%$; $p=0.08$ ), but there was no difference regarding RLS grading between the groups.

Conclusions and clinical implications. This study confirmed our previous findings in which the prevalence of RLS in patients with CHVS was significantly higher than in an age- and sex-matched healthy control group. However, we could not confirm the results of our prior study, where RLS was larger in CHVS than in CG. The tentative association between RLS and CHVS needs to be further examined.
\end{abstract}

Key words: chronic hyperventilation syndrome, right-to-left shunt, transcranial Doppler

\section{Introduction}

Chronic hyperventilation syndrome (CHVS) represents a frequent but poorly understood breathing pattern disorder that often goes undiagnosed due to its multi-systemic and apparently unrelated symptoms. CHVS can result in significant patient morbidity and an array of symptoms including breathlessness, chest tightness, dizziness, tremor, and paraesthesia; however, CHVS's underlying pathophysiology and effective management are not well understood [1]. CHVS is thought to be psychologically-based or physiologically-based. It involves breathing too deeply or too rapidly, resulting in hyperventilation which exceeds metabolic demands that lead to haemodynamic and chemical changes with characteristic clinical symptoms [2]. Approximately $6 \%$ of the general population, and $10 \%$ of patients in a general internal medicine practice, are reported to have CHVS. Patients with CHVS usually undergo extensive investigations, but in the majority of them, no organic causes can be found. Psychogenic hyperventilation is regarded as the main cause of CHVS; although CHVS and psychiatric disorders may overlap, only $25 \%$ of patients with CHVS have

Address for correspondence: Jacek Staszewski, Clinic of Neurology, Military Medical Institute, Warsaw, Poland, e-mail: jacekstaszewski@wp.pl 
panic attacks, and many patients with CHVS do not manifest low partial pressure of carbon dioxide ( $\mathrm{PaCO} 2)$ during attacks [3]. Different stressors such as emotional distress, but also pharmacological agents such as sodium lactate, caffeine, and isoproterenol, can provoke an exaggerated respiratory response.

We hypothesised that similarly to patients with migraines with aura or transient global amnesia, various endogenic substances might enter the systemic circulation in CHVS. These endogenous substances could enter through a cardiac or pulmonary right-to-left shunt (RLS) instead of being trapped in the pulmonary capillaries, and they might trigger or contribute to CHVS [5]. We have previously reported an increase in RLS frequency (40\% versus $8 \%$ ) through the patent foramen ovale (PFO) in 25 patients with CHVS when compared to 25 controls in a small unmatched case-control pilot study which assessed feasibility of the approach using contrast-enhanced transcranial Doppler (c-TCD) and transoesophageal echocardiography examinations (TEE) [4]. TEE is considered the gold standard for RLS diagnosis through PFO, but $\mathrm{c}-\mathrm{TCD}$ of the middle cerebral artery (MCA) has similar and high sensitivity $(70-100 \%)$, is relatively cheap, well tolerated, and feasible for larger studies [6]. TCD also allowed us to classify the grade of severity of RLS using a microembolic signals grading score [7]. The aim of the current study was two-fold: (1) to confirm our previous findings using a larger cohort of patients with CHVS, and (2) to evaluate the association between CHVS and the presence and grade of RLS using an individual matched case-control design with prospective data collection.

\section{Material and methods}

\section{Participants}

Consecutive patients with previously diagnosed CHVS were recruited from the neurological Outpatient Department between 2012 and 2016 and prospectively included in the study. CHVS was recognised in patients with typical recurrent clinical symptoms (dizziness, numbness, paresthesia, and/or near syncope) reproduced by voluntary hyperventilation and confirmed by the presence of spontaneous electromyographic activity with $\geq 2$ multiplets during provocative ischaemia and hyperventilation [8]. Subjects with asthma or other recognised pulmonary, cardiac, cerebrovascular, and/or psychiatric diseases were excluded. All patients with CHVS underwent psychiatric and cardiological consultations and had undergone brain neuroimaging (magnetic resonance imaging), EEG, carotid duplex ultrasonography, and TCD to exclude mental disorders and organic causes of the symptoms. Total and ionised calcium values were within normal reference range levels in all examined patients. The control group (CG) consisted of healthy volunteers matched with case subjects based on age and sex.

\section{Ultrasound examination}

Vascular RLS was diagnosed using c-TCD (Nicolet Companion III, Viasys) of the MCA to detect the presence of microbubble emboli (MB) following the standardised protocol recommended by the International Consensus Criteria (Fig. 1) [7]. All patients had an adequate temporal window for performing a TCD examination. The appearance of at least one contrast-induced MB signal on the c-TCD trace was regarded as pathognomonic for RLS. Patients were prepared using an 18-gauge needle inserted into the cubital vein and were examined in the supine position. The Doppler signal indicating blood flow in the single MCA was located at a depth of 45 to $65 \mathrm{~mm}$. The contrast agent was prepared using $9 \mathrm{ml}$ isotonic saline solution and $1 \mathrm{ml}$ air, which were then mixed with a three-way stopcock by exchange of the saline/air mixture between the syringes and injected as a bolus. The same physician prepared and injected the contrast into each patient. The MB were recorded with c-TCD at rest, and in case of no detection of MB in the MCA under basal conditions, the examination was repeated $5 \mathrm{sec}$ post injection following the Valsalva manoeuvre (VM) with controlled duration $(10 \mathrm{sec})$ and pressure (forced expiration against a manometer to $40 \mathrm{mmHg}$ ). The strength of the $\mathrm{VM}$ was considered sufficient when the MCA flow velocity amplitude decreased by $25 \%$. Before the test, patients were asked to practice a standardised VM. All examinations were done using a single experienced operator who was blinded to the subject diagnosis. All examination data were stored internally and analysed offline afterwards. Grading or RLS was performed by counting the number of embolic tracks on the power $\mathrm{M}$-mode and Doppler spectrogram in real time and offline. A four-level categorisation according to the MB count was applied: (1) $0 \mathrm{MB}$ (negative result); (2) 1-10 MB (low-grade shunt); (3) > $10 \mathrm{MB}$ and no curtain (medium-grade), and (4) curtain (large-grade) [7]. A curtain refers to a shower of $\mathrm{MB}$ in which a single bubble cannot be identified. RLS was considered permanent if it occurred during rest, and latent only if it occurred after a VM.

We evaluated the prevalence of RLS in addition to the shunt magnitude and permanent and latent RLS distributions in the study sample. We also compared migraine and migraine with aura frequencies in both studied groups. A migraine was diagnosed according to the International Classification of Headache Disorders, $3^{\text {rd }}$ edition [9]. Quantitative and qualitative demographic characteristics were summarised, and the data tabulated and tested for normality using the Shapiro-Wilk test. Categorical data are presented as frequencies and were compared using the chi-squared or Fisher's exact tests in cases in which these tests were appropriate. Continuous data were reported as means \pm standard deviations (SDs) and were compared using paired t-tests, while non-normal data were analysed using non-parametric tests. All statistical tests were two-sided, and $\mathrm{P}<0.05$ was considered statistically significant. 
All analyses were performed using Statistica 17 software (StatSoft Inc, USA).

This study complied with the tenets of the Declaration of Helsinki. The protocol of the study was accepted by the local ethics committee (Komisja Bioetyczna Wojskowego Instytutu Medycznego w Warszawie). All subjects provided written informed consent before any study-related procedures were performed.

\section{Results}

A total of 100 individuals (mean age $34 \pm 6$ years; females $80 \%$ ), including 50 with CHVS and 50 from the age- and sex-matched CG, were included in the analysis. The most common symptom in the CHVS group was vertigo or dizziness $(66 \%)$ followed by paraesthesia (40\%). The prevalence of migraines and migraines with aura was similar in both groups (Table 1). RLS was diagnosed in a significantly higher proportion of patients with CHVS (46\%) than in controls (16\%) (Table 2). There was a trend toward a higher proportion of permanent RLS in CHVS patients than in control subjects

Table 1. Baseline characteristics of the studied groups

\begin{tabular}{lccc} 
Group & CHVS & Control & P \\
N (\%) & 50 & 50 & \\
Mean age $( \pm S D)$ years & $33 \pm 7$ & $35 \pm 9$ & 0.7 \\
Females & $40(80)$ & $40(80)$ & 1 \\
Vertigo & $19(38)$ & 0 & - \\
Dizziness & $14(28)$ & 0 & - \\
Paraesthesia & $20(40)$ & 0 & - \\
Near syncope & $17(34)$ & 0 & - \\
Any migraine & $14(28)$ & $9(18)$ & 0.23 \\
Migraine with aura & $11(22)$ & $5(10)$ & 0.09 \\
\hline CHvs - chronichyperventilation syndrome &
\end{tabular}

Table 2. Right-to-left shunt in CHVS and control patients

$\begin{array}{lccc}\text { Group } & \text { CHVS } & \text { Control } & \text { P } \\ \text { N (\%) } & 50 & 50 & \\ \text { RLS in C-TCD } & 23(46) & 8(16) & <0.01 \\ \text { Permanent RLS } & 14(60) & 2(25) & 0.08 \\ \quad \text { Grade of RLS } & & & 0.4 \\ 1-10 \text { MB } & 6(26) & 5(63) & \\ 11-25 \text { MB } & 12(52) & 2(25) & \\ >25 \text { or curtain } & 5(22) & 1(12) & \\ \text { Migraine with aura } & 9(39) & 4(50) & 0.13\end{array}$

CHVS - chronic hyperventilation syndrome; RLS - right-to-left shunt; c-TCD - contrast transcranial Doppler; MB - microbubble emboli
( $60 \%$ versus $25 \%$; $\mathrm{p}=0.08$ ), but the grade of RLS did not differ between groups. Among all subjects with RLS, 13 (42\%) had concomitant migraines with aura (nine with CHVS and four with CG), but the difference between CHVS and CG was not statistically significant.

\section{Discussion}

This study confirmed our previous findings and revealed a higher RLS prevalence in CHVS patients (46\%) than in ageand sex-matched controls (16\%). We also demonstrated that patients with CHVS tended to have more frequent permanent shunts than controls. This was a new finding, but we did not find any differences regarding RLS grading between groups. RLS aetiology in these patients was probably due to PFO, since other findings such as intrapulmonary or other intracardiac RLS are rare.

The underlying mechanism by which some patients develop hyperventilation syndrome is unknown. The incidence of CHVS is higher in first-degree relatives than in the general population, but no clear genetic factors have been identified. CHVS often represents a simple manifestation of anxiety, rarely related to endocrine and/or respiratory diseases (such as hypoparathyroidism, asthma, pulmonary embolism) or central nervous system disorders (such as brainstem lesions); however, other patients manifest an abnormal respiratory response to sodium lactate and other chemical and emotional triggers, which result in excess minute ventilation and hypocarbia. Inducing a decrease in $\mathrm{PaCO} 2$ through voluntary hyperventilation may provoke CHVS in some, but not all, patients [10].

The pathogenetic role of RLS is also unknown, and as far as we know the association between RLS and CHVS has not yet been reported. PFO represents one of the main causes of cardiac RLS. Findings from autopsies or population-based studies have shown that $\mathrm{PFO}$ prevalence in the general population ranges from $10 \%$ to $29 \%$ [11]. PFO has been linked to paradoxical embolisation of thrombi and/or other microparticles or vasoactive chemicals leading to cryptogenic stroke, and also a broad spectrum of neurological diseases (e.g. migraines or migraines with aura, transient global amnesia, cluster headaches, and decompression sickness in sport divers) [12-13]. The prevalence of both cardiac and pulmonary RLS is especially high in patients with migraines with aura. Anzola et al. reported in a TCD study that RLS was present in $48 \%$ of individuals with migraines with aura, compared to $20 \%$ of healthy controls and $23 \%$ of patients with migraines without aura [14]. Migraines were also more prevalent in patients with an RLS at rest compared to those with a provocable RLS, and RLS grade was larger in migraine patients than in controls [15-16]. In our cohort, we observed that $42 \%$ of subjects with RLS suffered from migraines with aura, but there was no significant difference between CHVS and control subjects. On the other hand, there was a trend toward a higher prevalence of 


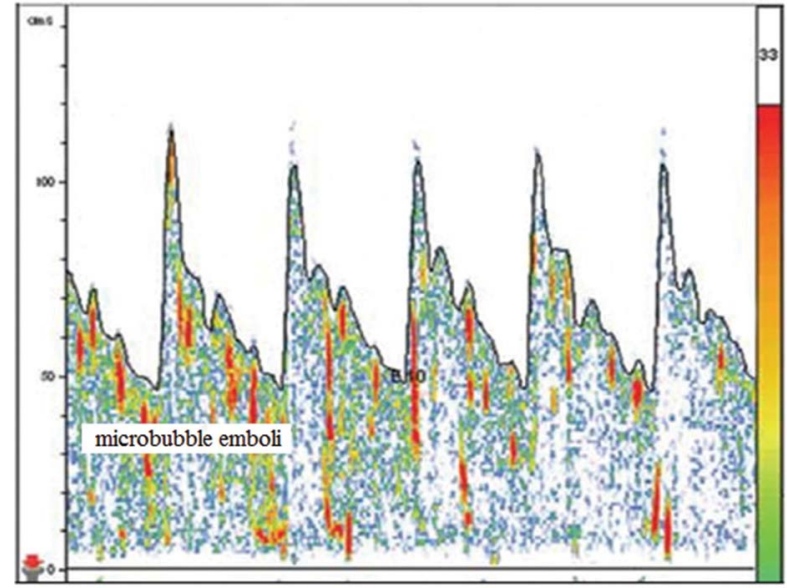

Figure 1. Transcranial Doppler recording showing multiple microbubbles

migraines with aura in patients with CHVD than in controls, which is in line with some previous reports showing a typical picture of CHVS in several patients with migraines at a headache phase [17-18].

Our study suggests an association between CHVS and RLS, but a causal relationship between these conditions remains speculative. Although we found more frequent permanent shunts in CHVS patients than in the CG, as we have previously reported, we did not demonstrate any differences in RLS grading between groups in the present study. As postulated in previous reports, RLS may allow venous-circulation of vasoactive chemicals that bypass the pulmonary filter and reach the cerebral circulation to induce a migraine and possible hyperventilation attack. But if this is so, the precise trigger has yet to be identified [19]. Whether or not chemical shunts or transient hypoxemia due to shunting of blood through the PFO plays a role in some patients with CHVS is unknown; however, CHVS is obviously related to a variety of mechanisms that may not be associated with hyperventilation alone [20]. While this putative mechanism accounts for some of the CHVS cases, it cannot account for all of them because not all CHVS patients have a shunt, and not all patients with an RLS have CHVS.

Our study has a number of limitations. It was a single-centre study with a limited number of patients. RLS was not confirmed by TEE, and we could not discriminate between RLS at the cardiac or pulmonary level based solely on c-TCD examination. In our previous report, we found extracardiac shunting via pulmonary arterio-venous malformation (AVM) in two patients with CHVS and none in the CG. This was an important finding because it suggests that the association of RLS with CHVD exists independently of shunt anatomy. We did not perform TEE or chest CT to diagnose extracardiac shunts because both procedures are invasive examinations, and they would possibly have had negative effects on recruitment. However, AVM in the general population is uncommon. In an autopsy study, only three pulmonary AVM cases were detected out of 15,000 consecutive autopsies [21]. TCD is considerably less invasive for the patient, and the VM, which significantly increases $\mathrm{MB}$ appearance, can be performed more accurately than TEE. Another limitation was the evaluation of a highly selective group of patients with CHVS. Patients with psychiatric or pulmonary diseases, frequently found in patients with CHVS, were excluded; thus, the study population may not be representative of the general CHVS patient population.

On the other hand, our study has some major advantages. The present case-control study is one of the first to address the prevalence of RLS in CHVS. We enrolled a relatively large number of well-characterised patients with rarely studied CHVS and compared them to age- and sex-matched controls. This is an important difference compared to the previous unmatched study because PFO prevalence and size may vary with age and sex, and also CHVS most commonly occurs among young women [22-24]. Although the age and sex distribution of this sample was comparable to the previously enrolled cohort, our current study design improved the efficiency of analysis by better distributing cases and controls between strata [25]. Another advantage is that all subjects had excluded cardiac and cerebrovascular diseases, and tests to detect RLS were performed in a blinded manner.

The reported association between CHVS and RLS is novel and difficult to explain, but whether it is functional or aetiological, it may improve the understanding of these conditions.

\section{Conclusions}

Our study suggests a possible link between RLS and CHVS. The prevalence of RLS in patients with CHVS was higher than in controls, but there was no difference between RLS grading or prevalence of migraine between groups. The clinical implications of these findings need to be determined.

\section{References}

1. Kern B, Rosh AJ. „Hyperventilation Syndrome.” eMedicine. Eds. Ryland P Byrd, et al. 28 Nov. 2016. Medscape. 01 Sep. 2011. http://emedicine.com/emerg/topic270.htm.

2. Jones M, Harvey A, Marston L, et al. Breathing exercises for dysfunctional breathing/hyperventilation syndrome in adults. Cochrane Database Syst Rev. 2013(5): CD009041, doi: 10.1002/14651858. CD009041.pub2, indexed in Pubmed: 23728685.

3. Boulding R, Stacey R, Niven R, et al. Dysfunctional breathing: a review of the literature and proposal for classification. Eur Respir Rev. 2016; 25(141): 287-294, doi: 10.1183/16000617.0088-2015, indexed in Pubmed: 27581828.

4. Staszewski J, Tomczykiewicz K, Brodacki B, et al. An increased frequency of right-to-left shunt in patients with chronic hyperventilation syndrome. Perspectives in Medicine. 2012; 1(1-12): 241-243, doi: 10.1016/j.permed.2012.02.027.

5. Klötzsch C, Sliwka U, Berlit P, et al. An increased frequency of patent foramen ovale in patients with transient global amnesia. Analysis of 
53 consecutive patients. Arch Neurol. 1996; 53(6): 504-508, indexed in Pubmed: 8660151.

6. Blersch WK, Draganski BM, Holmer SR, et al. Transcranial duplex sonography in the detection of patent foramen ovale. Radiology. 2002; 225(3): 693-699, doi: 10.1148/radiol.2253011572, indexed in Pubmed: 12461247.

7. Jauss M, Zanette E. Detection of right-to-left shunt with ultrasound contrast agent and transcranial Doppler sonography. Cerebrovasc Dis. 2000; 10(6): 490-496, doi: 10.1159/000016119, indexed in Pubmed: 11070388.

8. Valls-Solé J, Montero J. Role of EMG evaluation in muscle hyperactivity syndromes. J Neurol. 2004; 251(3): 251-260, doi: 10.1007/ s00415-004-0380-9, indexed in Pubmed: 15015003.

9. Headache Classification Committee of the International Headache Society (IHS). The International Classification of Headache Disorders, 3rd edition (beta version). Cephalalgia. 2013; 33(9): 629-808, doi: 10.1177/0333102413485658, indexed in Pubmed: 23771276.

10. Hornsveld HK, Garssen B, Dop MJ, et al. Double-blind placebo-controlled study of the hyperventilation provocation test and the validity of the hyperventilation syndrome. Lancet. 1996; 348(9021): 154-158, indexed in Pubmed: 8684155.

11. Movsowitz C, Podolsky LA, Meyerowitz CB, et al. Patent foramen ovale: a nonfunctional embryological remnant or a potential cause of significant pathology? J Am Soc Echocardiogr. 1992; 5(3): 259-270, indexed in Pubmed: 1622617.

12. Homma S, Sacco RL. Patent foramen ovale and stroke. Circulation. 2005; 112(7): 1063-1072, doi: 10.1161/CIRCULATIONAHA.104.524371, indexed in Pubmed: 16103257.

13. Del Sette M, Angeli S, Leandri M, et al. Migraine with aura and right-to-left shunt on transcranial Doppler: a case-control study. Cerebrovasc Dis. 1998; 8(6): 327-330, doi: 10.1159/000015875, indexed in Pubmed: 9774749.

14. Anzola GP, Magoni M, Guindani M, et al. Potential source of cerebral embolism in migraine with aura: a transcranial Doppler study. Neurology. 1999; 52(8): 1622-1625, indexed in Pubmed: 10331688.
15. Blau JN, Dexter SL. Hyperventilation during migraine attacks. Br Med J. 1980; 280(6226): 1254, indexed in Pubmed: 7388493.

16. Razavi M, Razavi $B$, Fattal $D$, et al. Hemiplegic migraine induced by exertion. Arch Neurol. 2000; 57(9): 1363-1365, indexed in Pubmed: 10987906.

17. Wilmshurst PT, Nightingale S, Walsh KP, et al. Effect on migraine of closure of cardiac right-to-left shunts to prevent recurrence of decompression illness or stroke or for haemodynamic reasons. Lancet. 2000; 356(9242): 1648-1651, indexed in Pubmed: 11089825.

18. Jesurum JT, Fuller CJ, Velez CA, et al. Migraineurs with patent foramen ovale have larger right-to-left shunt despite similar atrial septal characteristics. J Headache Pain. 2007; 8(4): 209-216, doi: 10.1007/ s10194-007-0396-5, indexed in Pubmed: 17901924.

19. Kerut EK, Norfleet WT, Plotnick GD, et al. Patent foramen ovale: a review of associated conditions and the impact of physiological size. J Am Coll Cardiol. 2001; 38(3): 613-623, indexed in Pubmed: 11527606.

20. Gardner WN. The pathophysiology of hyperventilation disorders. Chest. 1996; 109(2): 516-534, indexed in Pubmed: 8620731.

21. SLOAN RD, COOLEY RN. Congenital pulmonary arteriovenous aneurysm. Am J Roentgenol Radium Ther Nucl Med. 1953; 70(2): 183-210, indexed in Pubmed: 13065567.

22. Nedeltchev $\mathrm{K}$, Wiedmer S, Schwerzmann M, et al. Sex differences in cryptogenic stroke with patent foramen ovale. Am Heart J. 2008; 156(3): 461-465, doi: 10.1016/j.ahj.2008.04.018, indexed in Pubmed: 18760126.

23. Hagen PT, Scholz DG, Edwards WD. Incidence and size of patent foramen ovale during the first 10 decades of life: an autopsy study of 965 normal hearts. Mayo Clin Proc. 1984; 59(1): 17-20, indexed in Pubmed: 6694427.

24. Pfortmueller CA, Pauchard-Neuwerth SE, Leichtle AB, et al. Primary Hyperventilation in the Emergency Department: A First Overview. PLoS One. 2015; 10(6): e0129562, doi: 10.1371/journal. pone.0129562, indexed in Pubmed: 26110771.

25. Pearce Neil. Analysis of matched case-control studies BMJ. 2016; 352: 1969. 